\title{
Clinical Faceoff: Where Are We Going With Femoral Stem Fixation in THA?
}

\author{
Joseph T. Moskal MD, Susan G. Capps PhD, C. Anderson Engh Jr MD, \\ Anders Troelsen MD, PhD, DMSc
}

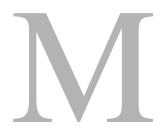
uch of the debate concerning cemented versus cementless versus hybrid THA centers on the THA construct, that is to say, on the combination of femoral stems and acetabular cups, not on individual components. There have been claims of a "North Atlantic Divide" regarding cemented, cementless, and hybrid THA with North America generally favoring cementless

Note from the Editor-in-Chief:

We are pleased to present to readers of Clinical Orthopaedics and Related Research ${ }^{\circledR}$ another installment of Clinical Faceoff, $a$ regular feature. This section is a pointcounterpoint discussion between recognized experts in their fields on a controversial topic. We welcome reader feedback on all of our columns and articles; please send your commentstoeic@clinorthop.org.

The authors certify that they, or any members of their immediate families, have no funding or commercial associations (consultancies, stock ownership, equity interest, patent/ licensing arrangements, etc.) that might pose a conflict of interest in connection with the submitted article.

All ICMJE Conflict of Interest Forms for authors and Clinical Orthopaedics and Related Research editors and board members are on file with the publication and can be viewed on request.

The opinions expressed are those of the writers and do not reflect the opinion or policy of $C O R R^{\circledR}$ or The Association of Bone and Joint Surgeons ${ }^{\circledR}$. fixation and Europe generally using cemented fixation [2, 7]. However, this is changing, as it appears that the use of cementless fixation is on the rise even outside the United States [11, 13]. Where are we going with femoral stem fixation in primary THA? What factors influence our decisions and choices in femoral stem fixation going forward?

The most likely avenues for answering these questions in an evidence-based manner might come from large-volume centers and registries. To that end, we have invited Dr. Andy Engh of the Anderson Orthopaedic Clinic to discuss the topic from the perspective of high-volume centers and Dr. Anders Troelsen of the

\section{J. T. Moskal MD ( $₫)$}

Institute for Orthopaedics and

Neurosciences, Carilion Clinic, 2331

Franklin Rd, SW, Roanoke, VA 24014 ,

USA

e-mail: jtmoskal@carilionclinic.org

S. G. Capps $\mathrm{PhD}$

BENSOL, Warsaw, IN, USA

C. A. Engh Jr MD

Anderson Orthopaedic Research

Institute, Alexandria, VA, USA

AndersTroelsen MD, PhD, DMSc

Department of Orthopaedic Surgery,

Copenhagen University Hospital

Hvidovre, Copenhagen, Denmark
Copenhagen University Hospital Hvidovre in Copenhagen, Denmark to discuss the topic from the registry perspective.

Joseph T. Moskal MD: We know that total hip replacement is generally successful; it is interesting to note that there are geographical differences in choice of fixation. What do you see regarding cemented versus cementless fixation? Why do you think it is so?

Andy Engh MD: While I believe reports involving only acetabular, femoral, or bearing surface results are potentially misleading to both clinicians and patients, I will focus on femoral fixation. We have reported 98\% 20-year Kaplan-Meier femoral survivorship with extensively porous coated cylindrical stems [1]. Other authors have reported 15- to 20-year femoral survivorship from $97 \%$ to $99 \%$ for some metaphyseal porous fixation or hydroxyapatite coated femoral designs [5, 6, 12]. Most of our arthroplasty fellows come to us having never used cemented femoral fixation for a THA. Additionally, the reason most North American orthopaedists prefer cementless fixation is because their generation has little or no cemented fixation training. We now know that both cemented and cementless femoral 


\section{Clinical Faceoff}

fixation are durable for the elderly patient, although cementless stems in the elderly are prone to early periprosthetic fractures. Registry reports are beginning to demonstrate that cementless femoral fixation clearly is more durable for the young patient [13]. Given the contrasting cemented and cementless femoral registry data for young compared to older patients, it would be reasonable to use a cementless stem in patients younger than 70 years of age and a cemented stem in older patients. In reality, surgeons and their staff find it easier to focus on one technique for the vast majority of their patients. In North America, that technique has been cementless femoral fixation. Lastly, patients have access to both good- and poor-quality information regarding THA, and are becoming more involved in their own implant selection. At my center, which is known for using cementless fixation, patients traditionally came to us for that reason, now the patients just assume we will use cementless rather than cemented fixation.

Anders Troelsen MD, PhD, DMSc: Although there are geographical differences in choice of fixation, with the highest percentage of uncemented fixation observed in North America, it is perhaps more noteworthy that increasing use of uncemented fixation is happening irrespective of geography. This trend is also observed in older patients-the group of patients with the best indication for use of cemented fixation. This is based on the finding of better durability of cemented implants compared with uncemented implants in elderly patients [11]. To add to the comments made by Dr. Engh, I believe we all face a challenge to make sure that cemented hip arthroplasty is not becoming a forgotten technique. The risk is that the outcome of cemented hip arthroplasty will be less favorable simply due to deteriorating surgeon skills in performing it. Lastly, we have to acknowledge that much of the "surgical behavior" in merging arthroplasty markets is driven by the "behavior" in North America and Europe. This is the time to go back to school and reinforce that our residents and fellows should become and remain good cement users.

Susan G. Capps PhD: Economics, both the cost of THA and the time that it takes to perform THA, has clearly become a driver. Do you believe that one method of fixation speaks more strongly to the economic concerns? Why?

Dr. Troelsen: It takes about 10 additional minutes to cement a femoral component. If saving this time leads to higher production (more procedures performed) then there could be an economic incentive to use cementless fixation. In many parts of the world, however, there are other variables deciding the number of arthroplasties that can be done, and thus the extra time spent may not play a role. I believe it is about choosing the mode of fixation with the highest chance of getting the patient a long-lasting, wellfunctioning hip, avoiding complications, and minimizing the likelihood of revision surgery. The cost of the implant varies across markets and countries, but assuming a uniform lower cost of a cemented implant, there could be an incentive to choose cemented fixation.

Avoiding complications and revisions are the keys, both for the sake of the patient and from the economic perspective. We have identified a high risk of periprosthetic fracture using an uncemented femoral implant in patients with Dorr Type C femurs, which is often found in older women. Registries report better survival of cemented fixation in elderly patients [11]. There is a good indication to use cemented femoral implants in elderly patients, and certainly in patients with Dorr Type $\mathrm{C}$ femurs. An algorithmic approach to use cemented fixation in patients with high risk of complications and revision surgery seems warranted.

Further, we have previously shown in our research [3] that the implant survival after revision surgery (with 


\section{Clinical Faceoff}

second revision as an endpoint) is better for primary THA that were cemented. Therefore, an economic incentive to increase the use of cemented fixation could even be evident beyond the first revision.

Dr. Engh: Current research does support a society-level economic benefit for the use of cemented or hybrid implants compared to cementless components [9]. However, the weaknesses of these studies warrants consideration. First, the premise of cementless fixation is that osseointegrated femoral components rarely fail by aseptic loosening and will likely last through a second decade of use. As registries accumulate data that span the entire second decade, I believe the economics of the comparison will change. Secondly, the data used for economic models utilize European registry data. These registries began accumulating data after the cemented fixation learning curve and at the beginning of the cementless learning curve. Arguably, the data used for these economic models are biased favoring cemented fixation. As of this writing, we have identified cementless stems that have excellent reproducible results across the "field" of surgeons and patients. Lastly, we have seen a gradual decrease in the average age of patients at the time of primary total hip replacement. If this trend continues, more and more patients will need the durability associated with osseointegrated cementless fixation.

Dr. Moskal: We know that the "geographic paradox," where the United States uses predominately cementless fixation in THA and Europe uses predominantly cemented fixation in THA, exists. What do you believe is the role of evidence-based medicine and/or registry data in promulgating this?

Dr. Engh: I disagree with the existence of a "geographic paradox" for femoral fixation. Yes, historically, surgeons from North America used cementless and Europe used cemented fixation. In addition, European registries traditionally reported better results with cemented than cementless fixation. It should be noted that THA has been a life-changing procedure for more than 50 years, yet data from national registries has only been available for 10 to 15 years. We tell our older patients that their hip should last as long as they need it, and our younger patients that their hip will probably last 20 years. We are able to make these statements because the accrual of 10-year cemented and cementless registry data allows analysis of confounding variables such as age, bearing surface, hybrid fixation, and periprosthetic fracture, which influence the comparison of cemented to cementless femoral fixation. There is a worldwide trend suggesting an increased use of cementless femoral fixation. Recent registry data [4] report revision for aseptic loosening of cementless femoral components is equal to that of cemented fixation. The main headline of the 2015 National Joint Registry of for England, Wales, Northern Ireland, and the Isle of Man [8] is "The debate regarding fixation as an isolated observation seems to become less of an issue in that it is the combination of the fixation, articulation and patient characteristics which influence the revision outcome" [8]. As cementless femoral fixation technique and implant designs improve, and the registries accumulate more data on cementless femoral fixation, the "geographic paradox" will become less evident than in the past.

Randomized clinical trials will always suffer from smaller sample size, performance bias (they are done at high-volume centers), shorter followup, and limited implant selection; however, randomized clinical trials provide the initial look at individual implant and technique results. In this role, randomized clinical trials provide the initial safety and performance check for an implant prior to general release.

Dr. Troelsen: The strength of registry data lies in the aggregation and analysis of performance data from surgeons in a wide variety of settings; 


\section{Clinical Faceoff}

this provides a more realistic, generalizable estimate of the performance in everyday use. This is helpful when surgeons, departments, and societies make decisions on best practices outside the setting of highly specialized units. Experts, from highly specialized units, can make most things work well (performance bias). I agree with Dr. Engh that randomized clinical trials are better (and absolutely needed) and more useful in showing efficacy or full potential of a treatment, but we should be aware that the observed efficacy or performance might not sustain when broadly introduced in everyday practice.

Dr. Capps: Our observation is that orthopaedic surgeons usually choose fixation techniques based on personal preference (experience or comfort or familiarity). Do you believe that these factors are appropriate drivers for fixation choice in THA? Why? Dr. Troelsen: Choices in orthopaedic treatment in all subspecialties are often based on personal preference and/or local treatment culture and history. There is evidence [10] suggesting better functional outcomes for patients if the surgeon does what he/she is most familiar and confident with. Therefore, preference or familiarity is an appropriate driver in the attempt to obtain good and reproducible results. However, if there is evidence that another technique, than the one preferred by the surgeon, may be superior, the surgeon has to reconsider the practice. In reality, there is a risk that cementing is becoming a forgotten technique and it is about time that we acknowledge that. That such a scenario has become reality is supported by the fact that a world-wide increased use of uncemented fixation has been observed, also in the elderly-a development that is paradoxical to the survivorship reports for cemented fixation in the elderly [11].

Dr. Engh: There is no question that evidence-based medicine and its associated-value equations are the current worldwide healthcare drivers. Orthopaedic surgeons and our patients, cannot afford to make decisions based on personal experience, comfort, or familiarity. Yes, THA is a life-changing procedure that has undeniable value for society. But we must continue to refine this procedure based on the evidence-not individual experience, marketing, or the media. In the past, surgeons have responded to problems with hip arthroplasty by improving polyethylene, becoming more efficient performing surgery, or by stopping use of poor-performing implants. If future data suggest strongly that cemented implants are superior, it will happen because the technique is easy and reproducible. In that event, surgeons will quickly relearn how to use cement.
Currently, there is no requirement that we adhere to a single-fixation approach if more than one is supported by evidence; in this case, there is room for more than one standard.

Dr. Moskal: Is it too simplistic to ask whether cementless is "better" than cemented (or vice versa); should we be asking more-nuanced questions here? If so, what are they, and how might we begin to answer them?

Dr. Engh: It is too simplistic to ask a single question such as cemented versus cementless. Clearly, implant performance depends on patient, implant, surgeon, and hospital variables. We have moved past the era of comparing implants. Implants must have $90 \%$ to $95 \%$ survivorship at 10 years. We must embrace what matters to the patient. Complications, reoperations, patientreported outcomes, and the variables that affect them are our current focus. Inclusion and reporting of Level 2 and 3 data to regional and national registries will take us beyond the simple question of what implants have better survivorship. In North America, publications from administrative databases that include complications, readmissions, and reoperations are common. While important, these publications suffer from absence of data validation, short duration, and lack of patient-reported outcomes. National registries with funding, validation, prospective 


\section{Clinical Faceoff}

longitudinal Level 2 and 3 data, and a reporting mechanism will answer the questions that are important for patients and their doctors. The goal is to add patient-reported outcomes and complications to the revision data, allowing a comprehensive understanding of THA outcomes.

Dr. Troelsen: I agree that it is too simplistic to discuss what mode of fixation is best. As we move ahead, we should identify the subgroup of patients who will benefit the most from a given mode of fixation. An example is the identification of highly increased risk of periprosthetic fractures in patients with Dorr Type C femurs. Avoiding early revisions is crucial. Beyond avoiding revisions, patient-reported outcome, as pointed out by Dr. Engh, is an important source of data when judging best possible treatment. However, for mode of fixation, it is perhaps less likely that this will be important for the patientreported outcome, assuming successful implant positioning and restoration of biomechanics with both kinds of fixation. In the case of fixation, it is fundamental to avoid early revisions, because this will lead to not only reoperation but also inferior patient-reported outcomes in some cases. Again, it remains important that we as surgeons master the techniques necessary to offer patients the best possible treatment-and that treatment choices regarding mode of fixation in the future are not a result of us forgetting how to use cement.

\section{References}

1. Belmont PJ Jr, Powers CC, Beykirch SE, Hopper RH Jr, Engh CA Jr, Engh CA. Results of the anatomic medullary locking total hip arthroplasty at a minimum of twenty years. A concise follow-up of previous reports. J Bone Joint Surg Am. 2008;90:1524-1530.

2. Dunbar MJ. Cemented femoral fixation: The North Atlantic divide. Orthopedics. 2009;32.

3. Gromov K, Pedersen AB, Overgaard S, Gebuhr P, Malchau H, Troelsen A. Do rerevision rates differ after first-time revision of primary THA with a cemented and cementless femoral component? Clin Orthop Relat Res. 2015;473:3391-3398.

4. Hooper GJ, Rothwell AG, Stringer M, Frampton C. Revision following cemented and uncemented primary total hip replacement: A seven-year analysis from the New Zealand Joint Registry. J Bone Joint Surg Br. 2009;91:451-458.

5. Lombardi AV, Berend KR, Mallory TH, Skeels MD, Adams JB. Survivorship of 2000 tapered titanium porous plasma-sprayed femoral components. Clin Orthop Relat Res. 2009;467:146-154.

6. McLaughlin JR, Lee KR. Cementless total hip replacement using second-generation components: A
12- to 16-year follow-up. $J$ Bone Joint Surg Br. 2010;92:1636-1641.

7. Murray DW. Cemented femoral fixation: The North Atlantic divide. Orthopedics. 2011;34:e462-e463.

8. National Joint Registry. $12^{\text {th }}$ annual report. National joint registry for England, Wales, Northern Ireland, and the Isle of Man. Available at: http:// www.njrcentre.org.uk/njrcentre/Portals/0/Documents/England/Reports/ 12th\%20annual\%20report/NJR\%20 Online\%20Annual\%20Report $\% 20$ 2015.pdf. Accessed March 13, 2016.

9. Pennington M, Grieve R, Sekhon JS, Gregg P, Black N, van der Meulen JH. Cemented, cementless, and hybrid prostheses for total hip replacement: Cost effectiveness analysis. BMJ. 2013;346:f1026.

10. Ritter MA, Davis KE, Farris A, Keating EM, Faris PM. The surgeon's role in relative success of PCL-retaining and PCL-substituting total knee arthroplasty. HSS J. 2014;10:107-115.

11. Troelsen A, Malchau E, Sillesen N, Malchau H. A review of current fixation use and registry outcomes in total hip arthroplasty: The uncemented paradox. Clin Orthop Relat Res. 2013;417:2052-2059.

12. Vidalain J. Twenty-year results of the cementless Corail stem. Int Orthop. 2011;35:189-194.

13. Wyatt M, Hooper G, Frampton C, Rothwell A. Survival outcomes of cemented compared to uncemented stems in primary total hip replacement. World J Orthop. 2014;5:591596. 